\title{
Dietary Salt and Blood Pressure: Verdict is Clear, so why Any Debate?
}

\author{
${ }^{1}$ Norm RC Campbell, ${ }^{2}$ Francesco P Cappuccio
}

\begin{abstract}
Repeated reviews of the evidence to produce recommendations for dietary salt intake have been conducted by independent committees of national and international scientific and governmental organizations. These recommendations support reducing dietary salt to less than $6 \mathrm{gm} /$ day and many to less than $5 \mathrm{gm} /$ day. Nevertheless, there is controversy about recommendations to reduce dietary salt. This commentary discusses low quality research studies and commercial interests as sources of the controversy. Especially, research that assesses usual salt intake in individuals based on a single spontaneously voided (spot) urine sample is discussed as a weak research method prone to erroneous findings. Further, some investigators have altered scientific formula to make their data using spot urine samples appear more robust and made misleading and false statements about evidence relating to dietary salt. Counterintuitive findings based on studies that have used spot urine samples is frequently disregarded in expert committee review given the low quality evidence is incompatible with higher quality evidence which shows direct linear relationships between dietary salt, hypertension and cardiovascular disease in the general population.
\end{abstract}

Keywords: Blood pressure, Cardiovascular disease, Conflict of interest, Diet, Hypertension, Nutrition, Public health, Salt, Sodium.

How to cite this article: Campbell NRC, Cappuccio FP. Dietary Salt and Blood Pressure: Verdict is Clear, so why Any Debate? Hypertens J 2016;2(2):57-59.

Source of support: Nil

Conflict of interest: None

\section{INTRODUCTION}

High amount of salt/sodium in the diet is one of the major global health risks. The Global Burden of Disease Study estimates 3.7 million deaths, and

\footnotetext{
1,2Professor

${ }^{1}$ Department of Medicine, Community Health Sciences and Physiology and Pharmacology, O'Brien Institute of Public Health and Libin Cardiovascular Institute of Alberta, University of Calgary, Calgary, Alberta, Canada

${ }^{2}$ Department of Cardiovascular Medicine and Epidemiology University of Warwick, WHO Collaborating Centre, Warwick Medical School, Coventry, UK

Corresponding Author: Norm RC Campbell, Professor Department of Medicine, Community Health Sciences and Physiology and Pharmacology, O'Brien Institute of Public Health and Libin Cardiovascular Institute of Alberta, University of Calgary, Calgary, Alberta, Canada, Phone: +4032107961 e-mail: ncampbell@ucalgary.ca
}

74 million years of disability (DALYs) in 2013 as a result of excess dietary salt (Institute for Health Metrics and Evaluation, 2015 University of Washington, http:// vizhub.healthdata.org/gbd-compare/, accessed Feb 26 2016). Multiple comprehensive reviews have been conducted by independent national and international health and scientific organizations to develop dietary salt recommendations, with the most recent review being conducted to support the 2015 American Dietary recommendations. ${ }^{1}$ These recommendations almost universally recommend dietary salt to be $<6$ gm $(<$ sodium $2400 \mathrm{mg}$ )/day or even lower, with the World Health Organization recommending individuals consume less than 5 gm salt ( $2000 \mathrm{mg}$ sodium) per day. ${ }^{2}$ Table 1 indicates recommended nomenclature for describing dietary salt.

Consistent public health recommendations are based on extensive evidence of harm from excess dietary salt. Animal models show multiple harms from adding salt to that naturally occurring in food, including linear increases in blood pressure, inflammation, hypertension, direct vascular, cardiac, and renal damage that are independent of blood pressure, asthma, osteoporosis, promotion of cancer, stroke, heart failure, and premature death. ${ }^{2,3}$ Hunter-gatherer populations that do not have added salt in their diets have little to no hypertension or increase in blood pressure with age and invariably consume less than $2.5 \mathrm{~g}$ salt (1000 $\mathrm{mg}$ sodium) per day. ${ }^{4}$ The few huntergatherer populations with high natural sources of dietary salt (e.g., salt in drinking water) had increases in blood pressure and hypertension, and consumed more than $2.5 \mathrm{~g}$ salt (1000 mg sodium) per day. Based on randomized controlled trials of changes in dietary salt being directly associated with changes in blood pressure, it is estimated

Table 1: Recommended* terminology for describing dietary salt daily intake

\begin{tabular}{ll}
\hline Term & Dietary salt intake $\mathrm{g} /$ day \\
\hline Low intake & Not defined, but likely $<0.25$ \\
Normal ancestral level of sodium & $<2.5$ \\
intake & $<5.0$ \\
Recommended & $\geq 5.0-10$ \\
High & $>10-15$ \\
Very high & $>15$ \\
Extremely high &
\end{tabular}

*Adapted recommendations of the World Hypertension League, World Action on Salt and Health and the Australian Division of World Action on Salt and Health ${ }^{4}$ 
that over $30 \%$ of hypertension is attributed to increased dietary salt (i.e., excess dietary salt causes more than 300 million people to have hypertension globally). ${ }^{2} \mathrm{~A}$ meta-analysis of controlled trials and several metaanalyses of higher-quality cohort studies find increased dietary salt associated with increased cardiovascular disease. ${ }^{5-7}$ Given the multitude of mechanisms for harm, it is not surprising that gastric cancer, renal cell cancer, asthma, adiposity, multiple sclerosis, rheumatoid arthritis, migraine, aortic aneurysm, Meniere's disease, ovarian dysfunction, renal stones, and osteoporosis have been associated with excess dietary salt in human studies. ${ }^{2}$

Nevertheless, there is still controversy about reducing dietary salt. The controversy is in part due to the results of flawed studies and in part due to the lack of a definitive long-term randomized controlled trial of salt reduction and cardiovascular outcomes. However, such trials are missing in most public health interventions with much less controversy. Scientists strongly feel that large outcome clinical trials are required to have had more than 40 years to conduct them.

In this commentary, we provide several nonscientific sources of the controversy ${ }^{8}$ to raise further awareness of the covert actions that are taking place to block global implementation of one of the most cost-effective public health strategies of the century. The first is the resilient conduct and extensive promotion of low-quality research that is prone to erronous results. The second is potential and real commercial conflicts of interest. The third is the lack of content knowledge about salt/public health of dissenting scientists and the fourth is the promotion of out of context and often misleading information.

Low-quality research can usually be exposed by examining the study methodology. ${ }^{9}$ Criteria that have been used to identify high-quality research designs include: (1) Assessing usual dietary salt using a valid method for at least one day and preferably multiple days over the study duration, (2) a study duration of at least 1 month when assessing changes in blood pressure and at least 1 year for assessing cardiovascular outcomes, (3) cohort studies that exclude people with disease due to the high likelihood of reverse causality (sick people eat less and die more frequently), and (4) analyses in cohort studies that do not adjust for blood pressure when examining outcomes related to salt causing increased blood pressure. Nearly all the studies that meet these modest quality criteria support salt reduction and have adequate statistical power to show harmful effects of increased dietary salt. ${ }^{6,7}$ The conduct of low-quality research that cannot meet these modest quality criteria have the potential to undermine the public health effort to reduce dietary salt and has led to an international call for setting research standards and to the formation of an international consortium of health and scientific organizations to set those standards. ${ }^{10}$

One of the best indicators of low-quality research is the use of a single spot urine to predict long-term salt ingestion in individuals. Nearly all ingested salt is excreted within 6 hours and short-term sodium excretion is under tight regulation by a multitude of factors that predict cardiovascular events independent of dietary salt (e.g., renal function, dietary potassium, sympathetic activity, renin, angiotensin, and aldosterone). Further, the equations used to predict 24-hour urine sodium excretion from a spot sample have variables (e.g., age, sex, and creatinine) that predict cardiovascular disease and blood pressure independently of dietary salt. Salt intake varies greatly "between" individuals (in a population or between populations). More importantly, it varies even more "within" the same individual from day to day. Finally, within the same day, the excretion of sodium (used as a marker of salt intake) shows a diurnal variation and it is affected by factors, such as posture, sleep-wake cycle, and neuroendocrine diurnal changes. There is no valid scientific basis for an assessment of salt intake that lasts hours to reflect usual salt intake over a period of years. Thus, use of a single spot urine to predict an individual's long-term salt consumption is the "sine qua non" of low-quality research and a "sin" in salt research!

The Prospective Urban Rural Epidemiology (PURE) study is often cited as evidence that lowering dietary salt can cause harm, but also noted to have multiple weaknesses. ${ }^{8}$ Although based on a very large international cohort, the PURE study assessed the usual salt intake of individuals using a single morning spot urine sample and related the results to both blood pressure and cardiovascular outcomes. In assessing the association of the spot sample to 24-hour urine samples, the PURE study also had a multitude of issues that were likely to inflate the association reported. Fifty percent of the 24-hour urines collected for the validation study were reported to be incomplete. Many additional incomplete 24-hour urines were likely included because the investigators substantively altered (without overt disclosure) the published method of excluding incomplete urine samples. The published formula predicting 24-hour urine sodium from the spot sample had four errata. The investigators only published the association of the spot sample with the dependent 24-hour urine sample likely inflating the degree of association by including the spot urine sample within the 24-hour urine sample. When requested, the investigators did not disclose the association of the spot urine sodium with the independent 24-hour urine sodium. The national population blood pressures in the PURE study are also noted to be markedly different from those in some high-quality national surveys conducted 
at the same time. The concerns about the quality of the PURE study sodium and blood pressure results make them very difficult to interpret scientifically and hence the results should not impact public health recommendations to reduce dietary salt.

Low-quality research on dietary sodium research in our opinion should not be funded, conducted, or published and needs to be critiqued and discouraged during presentations. Yet, such research is becoming very frequent. Why? The ease of assessing sodium in a spot urine sample collected in studies not appropriately designed to address salt and its health effects encourages post-hoc analyses for studies not primarily designed to answer the question regarding salt. Clear examples are the PURE Study and the exploitation of datasets, such as Transcend and Ontarget. Further, there is a strong propensity of journals to publish weak controversial research to stimulate their citation index. The polarization of positions is reflected in journals and their editorial stances. Meeting organizers highlight controversial findings on dietary salt, and feature low-quality research. The situation is not much different from the efforts to reduce tobacco use with mainstream public health and science on the one side and the tobacco industry and a few dissenting scientists on the other. It is notable that several dissenting scientists have acted as paid consultants of the Salt Institute - the most vocal opponent to public health actions on salt representing over 40 salt producers and food and drink manufacturers over the past 40 years. At least one of them is also indicated to have been a court witness for the tobacco industry and was paid $\$ 450,000-\$ 500,000$ USD to claim there was inadequate scientific proof that tobacco caused cancer. The World Hypertension League has called for quality research and for scientific meeting organizers to have sessions on dietary salt that feature high-quality research, and the impact of financial conflict of interest and low-quality research on research findings. ${ }^{11}$ Scientists and clinicians should view the dissidents and the low-quality research they promote skeptically rather than creating situations that promote "false" equipoise.

Science is a quest for the truth and this can only be met by the conduct of rigorous carefully designed research. Much of the controversy generated about reducing dietary salt is based on commercial interests and low-quality research very akin to the early days of reducing tobacco use. The World Hypertension League with international partners oversee regularly updated systematic reviews of the literature on dietary salt to aid those interested in dietary salt to stay up to date and to protect the implementation of one of the most cost-effective and beneficial public health interventions across the world.

\section{REFERENCES}

1. Dietary Guidelines Advisory Committee. Scientific Report of the 2015 Dietary Guidelines Advisory Committee. Part D. Chapter 6: Cross-cutting topics of public health importance. The Dietary Guidelines Advisory Committee, USA; 15 A.D. Feb 2.

2. Campbell NR, Lackland DT, Niebylski ML. 2014 Dietary Salt Fact Sheet of the World Hypertension League, International Society of Hypertension, Pan American Health Organization Technical Advisory Group on Cardiovascular Disease Prevention through Dietary Salt Reduction, the World Health Organization Collaborating Centre on Population Salt Reduction, and World Action on Salt \& Health. J Clin Hypertens (Greenwich) 2015 Jan;17(1):7-9.

3. Meneton P, Jeunemaitre X, de Wardener HE, MacGregor GA. Links between dietary salt intake, renal salt handling, blood pressure, and cardiovascular diseases. Physiol Rev 2005 Apr;85(2):679-715.

4. Campbell NR, Correa-Rotter R, Cappuccio FP, Webster J, Lackland DT, Neal B, MacGregor GA. Proposed nomenclature for salt intake and for reductions in dietary salt. J Clin Hypertens (Greenwich) 2015 Apr;17(4):247-251.

5. Poggio R, Gutierrez L, Matta MG, Elorriaga N, Irazola V, Rubinstein A. Daily sodium consumption and CVD mortality in the general population: systematic review and metaanalysis of prospective studies. Public Health Nutr 2015 Mar;18(4):695-704.

6. Adler AJ, Taylor F, Martin N, Gottlieb S, Taylor RS, Ebrahim S. Reduced dietary salt for the prevention of cardiovascular disease. Cochrane Database Syst Rev 2014;12:CD009217.

7. Aburto NJ, Ziolkovska A, Hooper L, Elliott P, Cappuccio FP, Meerpohl JJ. Effect of lower sodium intake on health: systematic review and meta-analyses. BMJ 2013;346:f1326.

8. Campbell NR, Lackland DT, MacGregor GA. Dietary sodium: a perspective on recent sodium evidence - its interpretation and controversies. J Clin Hypertens (Greenwich) 2013 Nov;15(11):765-768.

9. Cobb LK, Anderson CA, Elliott P, Hu FB, Liu K, Neaton JD, Whelton PK, Woodward M, Appel LJ, American Heart Association Council on Lifestyle and Metabolic Health. Methodological issues in cohort studies that relate sodium intake to cardiovascular disease outcomes: a science advisory from the American Heart Association. Circulation 2014 Mar;129(10):1173-1186.

10. Campbell NR, Appel LJ, Cappuccio FP, Correa-Rotter R, Hankey GJ, Lackland DT, MacGregor G, Neal B, Niebylski ML, Webster J, et al. A call for quality research on salt intake and health: from the World Hypertension League and supporting organizations. J Clin Hypertens 2014 Jul 16;16(7):469-471.

11. Campbell NRC, Lackland DT, Lisheng L, Zhang X-H, Nilsson PM, Niebylski ML; World Hypertension League Executive. The World Hypertension League: where now and where to in salt reduction. Cardiovasc Drugs Ther 2015 Jun;5(3):238-242. 